\title{
Reproducing Kernel Method for Solving Nonlinear Fractional Fredholm Integrodifferential Equation
}

\author{
Bothayna S. H. Kashkari (iD) ${ }^{1}$ and Muhammed I. Syam (iD) ${ }^{2}$ \\ ${ }^{1}$ Department of Mathematics, Faculty of Science, AL Faisaliah, King Abdulaziz University, Jeddah, Saudi Arabia \\ ${ }^{2}$ Department of Mathematical Sciences, UAE University, College of Science, P.O. Box 17551, Al-Ain, UAE
}

Correspondence should be addressed to Muhammed I. Syam; m.syam@uaeu.ac.ae

Received 8 May 2018; Accepted 17 October 2018; Published 2 December 2018

Academic Editor: Eulalia Martínez

Copyright (c) 2018 Bothayna S. H. Kashkari and Muhammed I. Syam. This is an open access article distributed under the Creative Commons Attribution License, which permits unrestricted use, distribution, and reproduction in any medium, provided the original work is properly cited.

\begin{abstract}
This article is devoted to both theoretical and numerical studies of nonlinear fractional Fredholm integrodifferential equations. In this paper, we implement the reproducing kernel method (RKM) to approximate the solution of nonlinear fractional Fredholm integrodifferential equations. Numerical results demonstrate the accuracy of the present algorithm. In addition, we prove the existence of the solution of the nonlinear fractional Fredholm integrodifferential equation. Uniformly convergence of the approximate solution produced by the RKM to the exact solution is proven.
\end{abstract}

\section{Introduction}

Fractional Fredholm integrodifferential equations have various applications in sciences and engineering. Most of these problems cannot be solved analytically, and hence finding accurate numerical solution for these problems will be very useful. Wazwaz $[1,2]$ studied the Fredholm integral equations of the form

$$
f(x)=r(x)+\lambda \int_{a}^{b} H(x, t) f(t) d t,
$$

where $a$ and $b$ are constants, $\lambda$ is a parameter, $r(x)$ is the data function, $H(x, t)$ is the kernel of the integral equation, and $f(x)$ is the unknown function that will determined. In this paper, we study the generalization of the above problem of the form

$$
D^{\alpha} f(x)=r(x)+\lambda \int_{a}^{b} H(x, t) f^{m}(t) d t, \quad 0<\alpha \leq 1
$$

subject to

$$
f(a)=a_{0} .
$$

Note that $D^{\alpha}$ in (2) is in the Caputo derivative. Equation (2) is called the nonlinear fractional Fredholm integrodifferential equations of the second kind characterized by the occurrence of the unknown function $u(x)$ inside and outside the integral sign. To homogenize the initial condition, we assume $q(x)=$ $f(x)-a_{0}$. Then,

$$
D^{\alpha} q(x)=r(x)+\lambda \int_{a}^{b} H(x, t)\left(q(t)+a_{0}\right)^{m} d t
$$

$$
0<\alpha \leq 1
$$

subject to

$$
q(a)=0
$$

In the following definition and theorem, we write the definition of Caputo derivative as well as the power rule which we are used in this paper. For more details on the geometric and physical interpretation for Caputo fractional derivatives, see [3]. 
Definition 1. For $m$ to be the smallest integer that exceeds $\alpha$, the Caputo fractional derivatives of order $\alpha>0$ is defined as

$$
\begin{aligned}
& D^{\alpha} q(x) \\
& = \begin{cases}\frac{1}{\Gamma(m-\alpha)} \int_{0}^{x}(x-\tau)^{m-\alpha-1} \frac{d^{m} q(\tau)}{d \tau^{m}} d \tau, & m-1<\alpha<m, \\
\frac{d^{m} q(x)}{d x^{m}}, & m \text { integer. }\end{cases}
\end{aligned}
$$

Theorem 2. The Caputo fractional derivative of the power function satisfies

$$
\begin{aligned}
& D^{\alpha} t^{s} \\
& \quad= \begin{cases}\frac{\Gamma(s+1)}{\Gamma(s-\alpha+1)} t^{p-s}, & n-1<s<n, s>n-1, \\
0, & s<\alpha .\end{cases}
\end{aligned}
$$

The reproducing kernel Hilbert space method is a useful numerical technique to solve nonlinear problems [4-6]. The reproducing kernel is given by this definition.

Definition 3. Let $M \neq \phi$. A function $K: A \times A \longrightarrow C$ is a kernel of $Z$ i if

(i) $H(., x) \in Z$ for all $x \in A$,

(ii) $(\phi(),. H(., x))=\phi(x)$ for all $x \in A$ and $\phi \in Z$.

The second condition is called the reproducing property and a Hilbert space which possesses a reproducing kernel is called a reproducing kernel Hilbert space (RKHS). More details can be in [7-14]. A description of the RKM for discretization of the linear fractional Fredholm integrodifferential equations problem (4)-(5) is presented in Section 2. In Section 3, we study the nonlinear fractional Fredholm integrodifferential equations. Several numerical examples and conclusions are discussed in Section 4. Conclusions and closing remarks are given in Section 5.

\section{Analysis of RKHSM for Linear Fractional Fredholm Integrodifferential Equations}

In this section, we discuss how to solve the following linear fractional Fredholm integrodifferential equation using RKHSM:

$$
D^{\zeta} q(x)=r(x)+\lambda \int_{a}^{b} H(x, t)\left(q(t)+a_{0}\right) d t
$$

$$
0<\zeta \leq 1
$$

subject to

$$
q(a)=0 .
$$

In order to solve problem (8)-(9), we construct the kernel Hilbert spaces $W_{2}^{1}[a, b]$ and $W_{2}^{2}[a, b]$ in which every function satisfy the boundary conditions (9). Let

$$
\begin{aligned}
& W_{2}^{2}[a, b]=\{f(s): f, \\
& \quad f^{\prime} \text { are absolutely continuous real value functions, } \\
& \left.\quad f^{\prime \prime} \in L^{2}[a, b], f(a)=0\right\} .
\end{aligned}
$$

The inner product in $W_{2}^{2}[a, b]$ is defined as

$$
(u(y), v(y))_{W_{2}^{2}[a, b]}=u(a) v(a)+\int_{a}^{b} u^{\prime}(y) v^{\prime}(y) d y
$$

and the norm $\|u\|_{W_{2}^{2}[a, b]}$ is given by

$$
\|u\|_{W_{2}^{2}[a, b]}=\sqrt{(u(y), u(y))_{W_{2}^{2}[a, b]}}
$$

where $u, v \in W_{2}^{2}[a, b]$.

Theorem 4. There exists $Q(s, y) \in W_{2}^{3}[0,1]$ such that, for any $u \in W_{2}^{2}[0,1]$ and each fixed $y, x \in[a, b]$, we have

$$
(u(y), Q(x, y))_{W_{2}^{2}[a, b]}=u(x) .
$$

In this case, $Q(x, y)$ is given by

$$
Q(x, y)=\left\{\begin{array}{ll}
\alpha_{0}(x)+\alpha_{1}(x) y, & y \leq x \\
\beta_{0}(x)+\beta_{1}(x) y, & y>x
\end{array}\right\}
$$

where

$$
\begin{aligned}
& \alpha_{0}(x)=\frac{a(b-x)}{a-b}, \\
& \alpha_{1}(x)=-\frac{b-x}{a-b}, \\
& \beta_{0}(x)=\frac{b(a-x)}{a-b}, \\
& \beta_{1}(x)=-\frac{a-x}{a-b} .
\end{aligned}
$$

Proof. Using the integration by parts, one can get

$$
\begin{aligned}
& (u(y), Q(x, y))_{W_{2}^{2}[a, b]} \\
& =u(a) Q(x, a)+u(b) Q_{y}(x, b)-u(a) Q_{y}(x, a) \\
& \quad-\int_{a}^{b} u(y) \frac{\partial^{2} Q}{\partial y^{2}}(x, y) d y .
\end{aligned}
$$

Since $u(y)$ and $Q(x, y) \in W_{2}^{2}[a, b]$,

$$
u(a)=0
$$

and

$$
Q(x, a)=0 .
$$


Thus,

$$
\begin{aligned}
& (u(y), Q(x, y))_{W_{2}^{2}[a, b]} \\
& \quad=u(b) Q_{y}(x, b)-\int_{a}^{b} u(y) \frac{\partial^{2} Q}{\partial y^{2}}(x, y) d y .
\end{aligned}
$$

Since $Q(x, y)$ is a reproducing kernel of $W_{2}^{2}[a, b]$,

$$
(u(y), Q(x, y))_{W_{2}^{2}[a, b]}=u(x) .
$$

Thus,

$$
\frac{\partial^{2} Q}{\partial y^{2}}(x, y)=\delta(y-x)
$$

where $\delta$ is the Dirac-delta function and

$$
Q_{y}(x, b)=0 .
$$

Since the characteristic equation of $\left(\partial^{2} \mathrm{Q} / \partial y^{2}\right)(x, y)=\delta(y-$ $x)$ is $\lambda^{2}=0$ and its characteristic value is $\lambda=0$ with 2 multiplicity roots, we write $Q(x, y)$ as

$$
Q(x, y)=\left\{\begin{array}{ll}
\alpha_{0}(x)+\alpha_{1}(x) y, & y \leq x \\
\beta_{0}(x)+\beta_{1}(x) y, & y>x
\end{array}\right\} .
$$

Since $\left(\partial^{2} \mathrm{Q} / \partial y^{2}\right)(x, y)=\delta(y-x)$, we have

$$
Q(x, x+0)=Q(x, x-0) .
$$

On the other hand, integrate $\left(\partial^{2} \mathrm{Q} / \partial y^{2}\right)(x, y)=\delta(y-x)$ from $x-\epsilon$ to $x+\epsilon$ with respect to $y$ and let $\epsilon \longrightarrow 0$ to get

$$
\frac{\partial Q}{\partial y}(x, x+0)-\frac{\partial Q}{\partial y}(x, x-0)=-1 .
$$

Using conditions (18) and (22)-(25), we get the following system of equations:

$$
\begin{aligned}
\alpha_{0}(x)+\alpha_{1}(x) a & =0, \\
\beta_{0}(x)+\beta_{1}(x) b & =0, \\
\alpha_{0}(x)+\alpha_{1}(x) x & =\beta_{0}(x)+\beta_{1}(x) x \\
\beta_{1}-c_{1} & =-1,
\end{aligned}
$$

We solved the last system using Mathematica to get

$$
\begin{aligned}
& \alpha_{0}(x)=\frac{a(b-x)}{a-b}, \\
& \alpha_{1}(x)=-\frac{b-x}{a-b}, \\
& \beta_{0}(x)=\frac{b(a-x)}{a-b}, \\
& \beta_{1}(x)=-\frac{a-x}{a-b} .
\end{aligned}
$$

Next, we study the space $W_{2}^{1}[a, b]$. Let

$W_{2}^{1}[a, b]=\{u(x):$

$u$ are absolutely continuous real value functions,

$\left.u^{\prime} \in L^{2}[a, b]\right\}$.

The inner product in $W_{2}^{1}[a, b]$ is defined as

$$
(u(y), v(y))_{W_{2}^{1}[a, b]}=u(a) v(a)
$$

$$
+\int_{a}^{b} u^{\prime}(y) v^{\prime}(y) d y
$$

and the norm $\|u\|_{W_{2}^{1}[a, b]}$ is given by

$$
\|u\|_{W_{2}^{1}[a, b]}=\sqrt{(u(y), u(y))_{W_{2}^{1}[a, b]}}
$$

where $u, v \in W_{2}^{1}[a, b]$.

Theorem 5. There exists $R(s, y) \in W_{2}^{1}[a, b]$ such that, for any $u \in W_{2}^{1}[a, b]$ and each fixed $y, x \in W_{2}^{1}[a, b]$, we have

$$
(u(y), R(x, y))_{W_{2}^{1}[a, b]}=u(x) .
$$

In this case, $R(x, y)$ is given by

$$
R(x, y)=\left\{\begin{array}{ll}
\alpha_{0}(x)+\alpha_{1}(x) y, & y \leq x \\
\beta_{0}(x)+\beta_{1}(x) y, & y>x
\end{array}\right\}
$$

where

$$
\begin{aligned}
& \alpha_{0}(x)=1-a, \\
& \alpha_{1}(x)=1, \\
& \beta_{0}(x)=1-a+x, \\
& \beta_{1}(x)=0 .
\end{aligned}
$$

Proof. Using the integration by parts, one can get

$$
\begin{aligned}
(u(y), R(s, y))_{W_{2}^{1}[a, b]} & \\
= & u(a) R(s, a)+\int_{a}^{b} u^{\prime}(y) \frac{\partial R}{\partial y}(s, y) d y \\
= & u(a) R(s, a)+u(b) \frac{\partial R}{\partial y}(s, b)-u(a) \frac{\partial R}{\partial y}(s, a) \\
& -\int_{a}^{b} u(y) \frac{\partial^{2} R}{\partial y^{2}}(s, y) d y .
\end{aligned}
$$

Since $R(x, y)$ is a reproducing kernel of $W_{2}^{1}[a, b]$,

$$
(u(y), R(x, y))_{W_{2}^{1}[a, b]}=u(x) .
$$

Then,

$$
-\frac{\partial^{2} R}{\partial y^{2}}(x, y)=\delta(y-x)
$$


and

$$
\begin{aligned}
R(x, a)-\frac{\partial R}{\partial y}(x, a) & =0, \\
\frac{\partial R}{\partial y}(x, b) & =0 .
\end{aligned}
$$

Since the characteristic equation of $-\left(\partial^{2} R / \partial y^{2}\right)(x, y)=\delta(y-$ $x)$ is $\lambda^{2}=0$ and its characteristic value is $\lambda=0$ with 2 multiplicity roots, we write $R(x, y)$ as

$$
R(x, y)=\left\{\begin{array}{ll}
\alpha_{0}(x)+\alpha_{1}(x) y, & y \leq x \\
\beta_{0}(x)+\beta_{1}(x) y, & y>x
\end{array}\right\} .
$$

Since $\left(\partial^{2} R / \partial y^{2}\right)(x, y)=-\delta(y-x)$, we have

$$
\begin{gathered}
R(x, x+0)-R(x, x-0)=0 \\
\frac{\partial R}{\partial y}(x, x+0)-\frac{\partial R}{\partial y}(x, x-0)=-1
\end{gathered}
$$

Hence,

$$
\begin{aligned}
\alpha_{0}(x)+\alpha_{1}(x) a-\alpha_{1}(x) & =0, \\
\beta_{1}(x) & =0, \\
\alpha_{0}(x)+\alpha_{1}(x) x & =\beta_{0}(x)+\beta_{1}(x) x, \\
d_{1}(x)-\alpha_{1}(x) & =-1 .
\end{aligned}
$$

Then,

$$
\begin{aligned}
& \alpha_{0}(x)=1-a, \\
& \alpha_{1}(x)=1, \\
& \beta_{0}(x)=1-a+x, \\
& \beta_{1}(x)=0 .
\end{aligned}
$$

Now, we present how to solve problem (8)-(9) using the reproducing kernel method. Let

$$
\sigma_{i}(x)=R\left(x_{i}, .\right)
$$

for $i \in N$. It is clear that $L: W_{2}^{2}[a, b] \longrightarrow W_{2}^{1}[a, b]$ is bounded. Let

$$
\psi_{i}(x)=L^{*} \sigma_{i}(x)
$$

where $L\left(\sigma_{i}(x)\right)=D^{\alpha} f(x)-\lambda \int_{0}^{b} K(x, t)\left(f(t)-a_{0}\right) d t$ and $L^{*}$ is the adjoint operator of $L$. Using Gram-Schmidt orthonormalization to generate orthonormal set of functions $\left\{\bar{\psi}_{i}(x)\right\}_{i=1}^{\infty}$ where

$$
\bar{\psi}_{i}(x)=\sum_{j=1}^{i} \alpha_{i j} \psi_{j}(x)
$$

and $\alpha_{i j}$ are coefficients of Gram-Schmidt orthonormalization. In the next theorem, we show the existence of the solution of Problem (8)-(9).
Theorem 6. If $\left\{x_{i}\right\}_{i=1}^{\infty}$ is dense on $[a, b]$, then

$$
f(x)=\sum_{i=1}^{\infty} \sum_{j=1}^{i} \alpha_{i j} g\left(x_{j}\right) \bar{\psi}_{i}(x) .
$$

Proof. First, we want to prove that $\left\{\psi_{i}(x)\right\}_{i=1}^{\infty}$ is the complete system of $W_{2}^{2}[a, b]$ and $\psi_{i}(x)=L\left(Q\left(x, x_{i}\right)\right)$. It is clear that $\psi_{i}(x) \in W_{2}^{2}[a, b]$ for $i \in N$. Simple calculations implies that

$$
\begin{aligned}
\psi_{i}(x) & =L^{*} \sigma_{i}(x)=\left(L^{*} \sigma_{i}(x), Q(x, y)\right)_{W_{2}^{3}[0,1]} \\
& =\left(\sigma_{i}(x), L(Q(x, y))\right)_{W_{2}^{2}[a, b]}=L\left(Q\left(x, x_{i}\right)\right) .
\end{aligned}
$$

For each fixed $f(x) \in W_{2}^{2}[a, b]$, let

$$
\left(f(x), \psi_{i}(x)\right)_{W_{2}^{2}[a, b]}=0, \quad i \in N .
$$

Then,

$$
\begin{aligned}
\left(f(x), \psi_{i}(x)\right)_{W_{2}^{2}[a, b]} & =\left(f(x), L^{*} \sigma_{i}(x)\right)_{W_{2}^{2}[a, b]} \\
& =\left(L f(x), \sigma_{i}(x)\right)_{W_{2}^{2}[a, b]} \\
& =\operatorname{Lf}\left(x_{i}\right)=0 .
\end{aligned}
$$

Since $\left\{x_{i}\right\}_{i=1}^{\infty}$ is dense on $[a, b], L f(x)=0$. Since $L^{-1}$ exists, $f(x)=0$. Thus, $\left\{\psi_{i}(x)\right\}_{i=1}^{\infty}$ is the complete system of $W_{2}^{2}[a, b]$. Second, we prove (47). Simple calculations imply that

$$
\begin{aligned}
q(x) & =\sum_{i=1}^{\infty}\left(q(x), \bar{\psi}_{i}(x)\right)_{W_{2}^{2}[a, b]} \bar{\psi}_{i}(x) \\
& =\sum_{i=1}^{\infty} \sum_{j=1}^{i} \alpha_{i j}\left(q(x), L^{*}\left(Q\left(x, x_{j}\right)\right)\right)_{W_{2}^{2}[a, b]} \bar{\psi}_{i}(x) \\
& =\sum_{i=1}^{\infty} \sum_{j=1}^{i} \alpha_{i j}\left(L q(x), Q\left(x, x_{j}\right)\right)_{W_{2}^{2}[a, b]} \bar{\psi}_{i}(x) \\
& =\sum_{i=1}^{\infty} \sum_{j=1}^{i} \alpha_{i j}\left(r(x), Q\left(x, x_{j}\right)\right)_{W_{2}^{2}[a, b]} \bar{\psi}_{i}(x) \\
& =\sum_{i=1}^{\infty} \sum_{j=1}^{i} \alpha_{i j} r\left(x_{j}\right) \bar{\psi}_{i}(x) .
\end{aligned}
$$
by

Let the approximate solution of problem (8)-(9) be given

$$
q_{N}(s)=\sum_{i=1}^{N} \sum_{j=1}^{i} \alpha_{i j} r\left(s_{j}\right) \bar{\psi}_{i}(s) .
$$

In the next theorem, we show the uniformly convergent of the $\left\{q_{N}(x)\right\}_{N=1}^{\infty}$ to $q(x)$.

Theorem 7. If $q(x)$ and $q_{N}(x)$ are given as in (47) and (52), then $\left\{q_{N}(x)\right\}_{N=1}^{\infty}$ converges uniformly to $q(x)$. 
Proof. For any $x \in[a, b]$,

$$
\begin{aligned}
& \left\|q(x)-q_{N}(x)\right\|_{W_{2}^{2}[a, b]}^{2}=\left(q(x)-q_{N}(x), q(x)\right. \\
& \left.-q_{N}(x)\right)_{W_{2}^{2}[a, b]}=\sum_{i=N+1}^{\infty}\left(\left(q(x), \bar{\psi}_{i}(x)\right)_{W_{2}^{2}[a, b]}\right. \\
& \left.\cdot \bar{\psi}_{i}(x),\left(q(x), \bar{\psi}_{i}(x)\right)_{W_{2}^{2}[a, b]} \bar{\psi}_{i}(x)\right)_{W_{2}^{2}[a, b]} \\
& =\sum_{i=N+1}^{\infty}\left(q(x), \bar{\psi}_{i}(x)\right)_{W_{2}^{2}[a, b]}^{2} .
\end{aligned}
$$

Hence,

$$
\begin{aligned}
& \sup _{x \in[a, b]}\left\|q(x)-q_{N}(x)\right\|_{W_{2}^{2}[a, b]}^{2} \\
& =\sup _{x \in[a, b]} \sum_{i=N+1}^{\infty}\left(q(x), \bar{\psi}_{i}(x)\right)_{W_{2}^{2}[a, b]}^{2} .
\end{aligned}
$$

From Theorem 6, one can see that $\sum_{i=1}^{\infty}\left(q(x), \bar{\psi}_{i}(x)\right)_{W_{2}^{2}[a, b]} \bar{\psi}_{i}(x)$ converges uniformly to $q(x)$. Hence,

$$
\sup _{x \in[a, b]}\left\|q(x)-q_{N}(x)\right\|_{W_{2}^{2}[a, b]}=0 \quad \text { as } N \longrightarrow \infty
$$

which implies that $\left\{q_{N}(x)\right\}_{N=1}^{\infty}$ converges uniformly to $q_{N}(x)$.

\section{Analysis of RKHSM for Nonlinear Fractional Fredholm Integrodifferential Equations}

In this section, we discuss how to solve the following the following problem using RKHSM:

$$
\begin{aligned}
D^{\zeta} q(x)=r(x)+\lambda \int_{a}^{b} h(x, t)\left(q(t)+a_{0}\right)^{m} d t & \\
& 0<\zeta \leq 1
\end{aligned}
$$

subject to

$$
q(a)=0
$$

Let

$$
G(x, q)=\lambda \int_{a}^{b} H(x, t)\left(q(t)+a_{0}\right)^{m} d t .
$$

We construct a homotopy as follows:

$$
H(q, p)=D^{\zeta} q(x)-r(x)-p G(x, q)=0
$$

where $p \in[0,1]$ is an embedding parameter. For $p=0$, we get a linear equation

$$
D^{\zeta} f q(x)-r(x)=0
$$

which can be solved by using RKHSM as we described in the pervious section. If $\lambda=1$, we turn out to be problem (56). Following the Homotopy Perturbation method [15], we expand the solution in terms of the Homotopy parameter $\lambda$ as

$$
q=q_{0}+p q_{1}+p^{2} q_{2}+p^{3} q_{3}+\cdots
$$

Substitute (61) into (59) and equate the coefficients of the identical powers of $\lambda$ to get the following system:

$$
\begin{gathered}
\lambda^{0}: D^{\zeta} q_{0}(x)=r(x), \quad q_{0}(a)=0, \\
\lambda^{1}: D^{\zeta} q_{1}(x)=\left.G\left(x, \sum_{i=0}^{\infty} p^{i} q_{i}\right)\right|_{p=0}, \quad q_{1}(a)=0, \\
\lambda^{2}: D^{\zeta} q_{2}(x)=\left.\frac{d G\left(x, \sum_{i=0}^{\infty} p^{i} q_{i}\right)}{d \lambda}\right|_{p=0}, \quad q_{2}(a)=0, \\
\lambda^{3}: D^{\zeta} q_{3}(x)=\left.\frac{d^{2} G\left(x, \sum_{i=0}^{\infty} p^{i} q_{i}\right)}{d \lambda^{2}}\right|_{p=0}, \quad q_{3}(a)=0, \\
\lambda^{k}: D^{\zeta} q_{k}(x)=\left.\frac{d^{k-1} G\left(x, \sum_{i=0}^{\infty} p^{i} q_{i}\right)}{d \lambda^{k-1}}\right|_{p=0},
\end{gathered}
$$

From (61), it is easy to see that the solution to problem (56)-(57) is given by

$$
q(x)=\sum_{k=0}^{\infty} q_{k}(x)=\sum_{k=0}^{\infty}\left(\sum_{i=1}^{\infty} \sum_{j=1}^{i} \alpha_{i j} r_{k}\left(x_{j}\right) \bar{\psi}_{i}(x)\right) .
$$

We approximate the solution of problem (56)-(57) by

$$
f_{N, M}(x)=\sum_{k=0}^{m}\left(\sum_{i=1}^{n} \sum_{j=1}^{i} \alpha_{i j} g_{k}\left(s_{j}\right) \bar{\psi}_{i}(x)\right) \text {. }
$$

\section{Numerical Results}

In this section, we present three numerical examples to show the efficiency of the proposed method.

Example 1. Consider the following fractional Fredholm integrodifferential equation:

$$
D^{1 / 2} u(x)=g(x)+\lambda \int_{0}^{1} x t u(t) d t
$$

subject to

$$
u(0)=0
$$

where $g(x)=(32 / 3 \sqrt{\pi}) x^{3 / 2}+(16 / \sqrt{\pi}) x^{5 / 2}-2 \lambda x$. Using $N=$ 10 , the approximate solution is

$$
u_{10}(x)=4 x^{2}+5 x^{3} .
$$

Thus, $u_{10}(x)$ is the exact solution. 


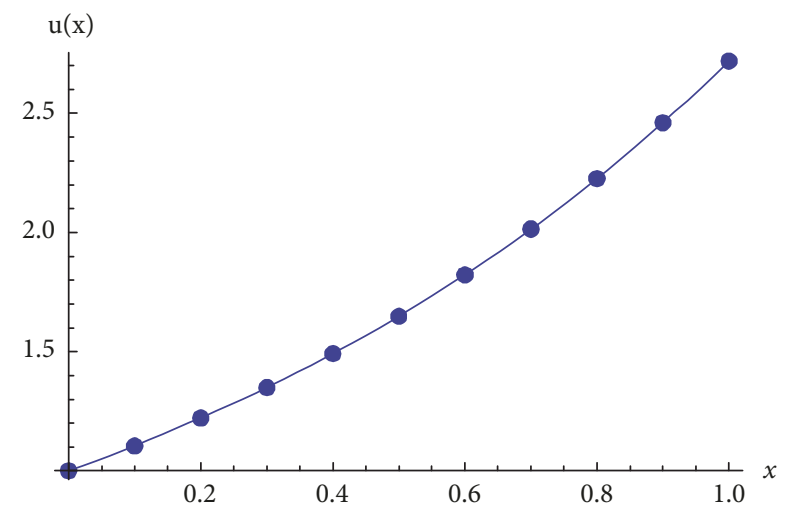

FIGURE 1: The exact and approximate solution for $N=15$.

TABLE 1: The error for Example 1.

\begin{tabular}{lc}
\hline$n$ & $e_{15}$ \\
\hline 10 & $2.8 * 10^{-10}$ \\
\hline 13 & $4.1 * 10^{-12}$ \\
\hline 16 & $3.9 * 10^{-13}$ \\
\hline
\end{tabular}

Example 2. Consider the following fractional Fredholm integrodifferential equation:

$$
D^{1 / 4} u(x)=g(x)+2 \int_{0}^{1} e^{x-t} u^{2}(t) d t
$$

subject to

$$
u(0)=1
$$

where $g(x)=x^{1 / 4} E_{1,7 / 2}(x)-2(e-1) e^{x}$ and $E_{a, b}(x)$ is the two-parameter of Mittag-Leffler function. The exact solution is $u(x)=e^{x}$. Using $N=15$, the approximate solution is given in Figure 1. The error

$$
e_{n}=\max \left\{\left|u(x)-u_{n}(x)\right|: x=0,0.1,0.2, \ldots, 1\right\}
$$

is given in Table 1.

Example 3. Consider the following fractional Fredholm integrodifferential equation:

$$
D^{3 / 4} u(x)=g(x)+\int_{0}^{1} e^{x} t u^{4}(t) d t
$$

subject to

$$
u(0)=2
$$

where $g(x)=(24 / \Gamma(17 / 4)) x^{13 / 4}-(10307 / 630) e^{x}$ and $\Gamma$ is the gamma function. The exact solution is $u(x)=e^{x}$. Using $N=$ 12 , the approximate solution is

$$
u_{12}(x)=x^{4}+2 .
$$

Hence, $u_{12}(x)$ is the exact solution.

\section{Conclusions and Closing Remarks}

In this paper, we investigate the nonlinear fractional Fredholm integrodifferential equations where $0<\alpha \leq 1$. We implement the reproducing kernel method to approximate the solution of the proposed problem. Numerical results demonstrate the accuracy of the present algorithm. In addition, we prove the existence of the solution of the nonlinear fractional Fredholm integrodifferential equation. Uniformly convergence of the approximate solution produced by the RKM to the exact solution is proven. We noted the following:

(i) The proposed method is very accurate. We get the exact solution in Examples 1 and 3.

(ii) Form Table 1, we note that the error is very small in Example 2.

(iii) Figure 1 shows that the approximate solution and the exact solution are identical.

(iv) The proposed method can be generalized for more models in Physics and Engineering.

\section{Data Availability}

The data and results used to support the findings of this study are included within the article.

\section{Conflicts of Interest}

The authors declare that they have no conflicts of interest.

\section{Acknowledgments}

This work was supported by the Deanship of Scientific Research (DSR), King Abdulaziz University, Jeddah, under Grant no. (D-001-363-1439). The authors, therefore, gratefully acknowledge the DSR technical and financial support.

\section{References}

[1] A.-M. Wazwaz, A First Course in Integral Equations, WSPC, New Jersey, NJ, USA, 1997.

[2] A.-M. Wazwaz, "The regularization method for Fredholm integral equations of the first kind," Computers \& Mathematics with Applications. An International Journal, vol. 61, no. 10, pp. 2981-2986, 2011.

[3] K. B. Oldham and J. Spanier, The Fractional Calculus, Academic Press, New York, NY, USA, 1974, reprinted in 2006.

[4] N. Aronszajn, "Theory of reproducing kernels," Transactions of the American Mathematical Society, vol. 68, pp. 337-404, 1950.

[5] E. Barbieri and M. Meo, "A fast object-oriented Matlab implementation of the reproducing kernel particle method," Computational Mechanics, vol. 49, no. 5, pp. 581-602, 2012.

[6] M. Cui and Y. Lin, Nonlinear Numerical Analysis in the Reproducing Kernel Space, Nova Science, New York, NY, USA, 2009.

[7] M. I. Syam and H. I. Siyyam, "An efficient technique for finding the eigenvalues of fourth-order Sturm-Liouville problems," Chaos, Solitons \& Fractals, vol. 39, no. 2, pp. 659-665, 2009. 
[8] B. S. Attili and M. I. Syam, "Efficient shooting method for solving two point boundary value problems," Chaos, Solitons \& Fractals, vol. 35, no. 5, pp. 895-903, 2008.

[9] M. F. El-Sayed and M. I. Syam, "Electrohydrodynamic instability of a dielectric compressible liquid sheet streaming into an ambient stationary compressible gas," Archive of Applied Mechanics, vol. 77, no. 9, pp. 613-626, 2007.

[10] B. S. Attili, K. Furati, and M. I. Syam, "An efficient implicit Runge-Kutta method for second order systems," Applied Mathematics and Computation, vol. 178, no. 2, pp. 229-238, 2006.

[11] M. I. Syam and H. I. Siyyam, "Numerical differentiation of implicitly defined curves," Journal of Computational and Applied Mathematics, vol. 108, no. 1-2, pp. 131-144, 1999.

[12] M. I. Syam, "The modified Broyden-variational method for solving nonlinear elliptic differential equations," Chaos, Solitons \& Fractals, vol. 32, no. 2, pp. 392-404, 2007.

[13] M. I. Syam and B. S. Attili, "Numerical solution of singularly perturbed fifth order two point boundary value problem," Applied Mathematics and Computation, vol. 170, no. 2, pp. 10851094, 2005.

[14] M. F. El-Sayed and M. I. Syam, "Numerical study for the electrified instability of viscoelastic cylindrical dielectric fluid film surrounded by a conducting gas," Physica A: Statistical Mechanics and its Applications, vol. 377, no. 2, pp. 381-400, 2007.

[15] P. D. Ariel, M. I. Syam, and Q. M. Al-Mdallal, "The extended homotopy perturbation method for the boundary layer flow due to a stretching sheet with partial slip," International Journal of Computer Mathematics, vol. 90, no. 9, pp. 1990-2002, 2013. 


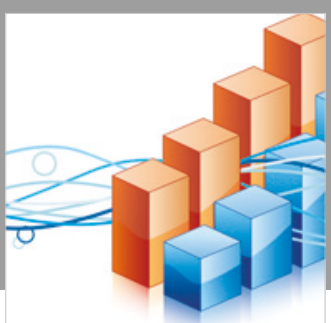

Advances in

Operations Research

\section{-n-m}
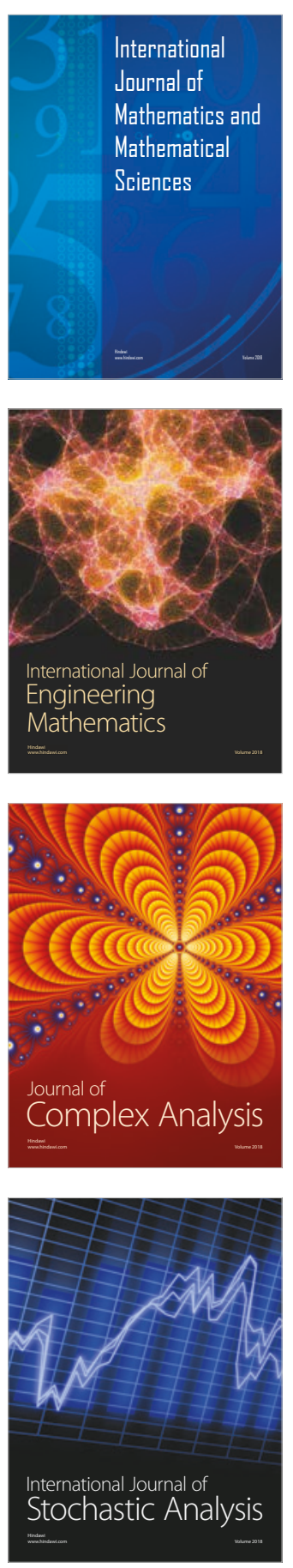
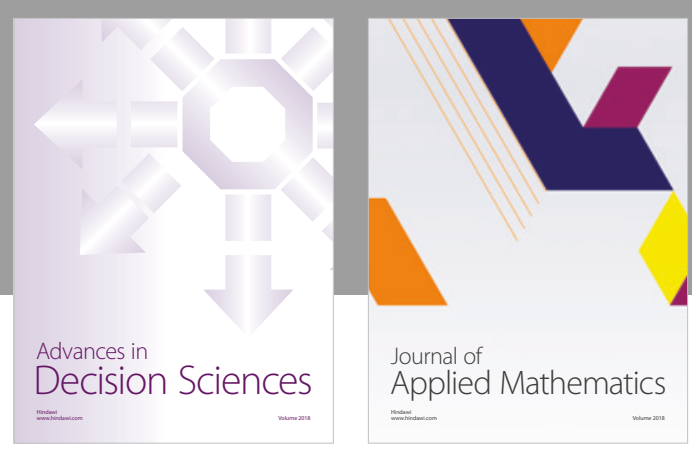

Journal of

Applied Mathematics
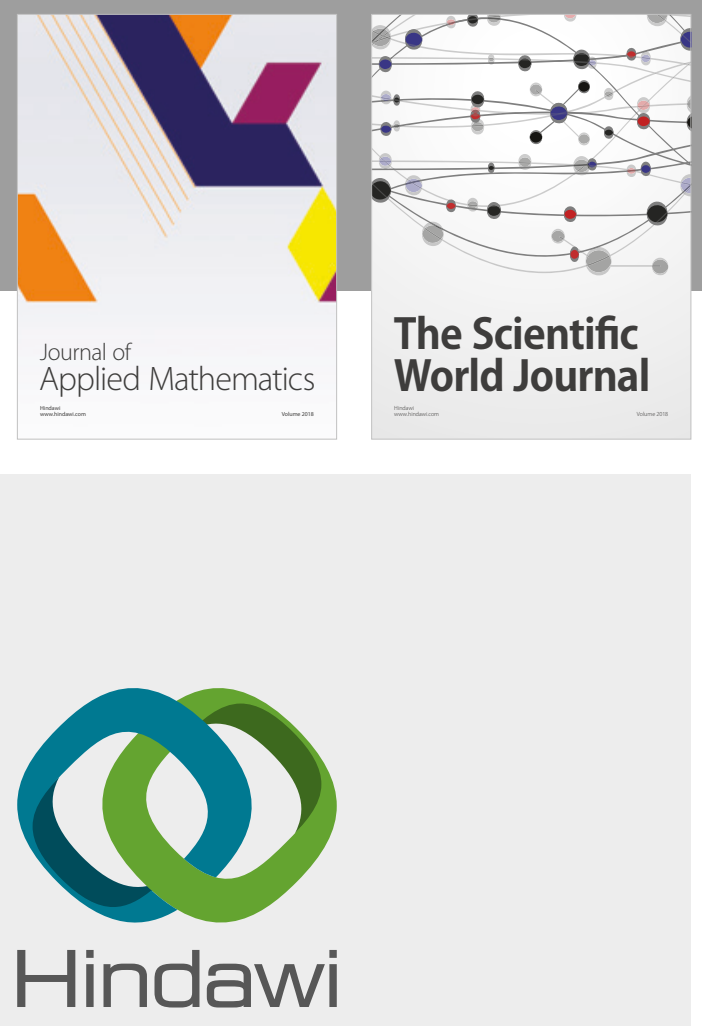

Submit your manuscripts at

www.hindawi.com

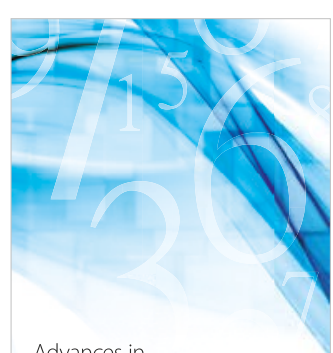

Advances in
Numerical Analysis
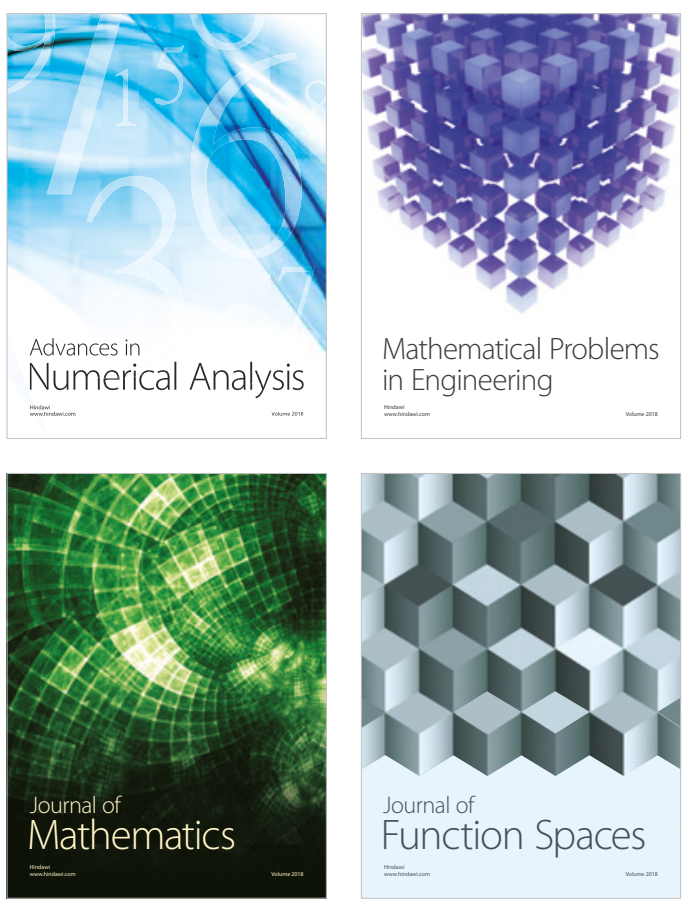

Mathematical Problems in Engineering

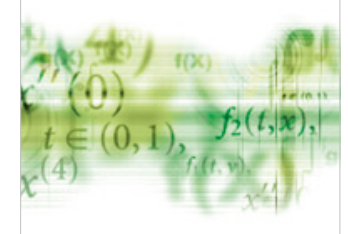

International Journal of

Differential Equations

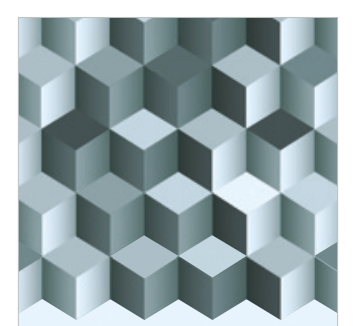

Journal of

Function Spaces
The Scientific

World Journal

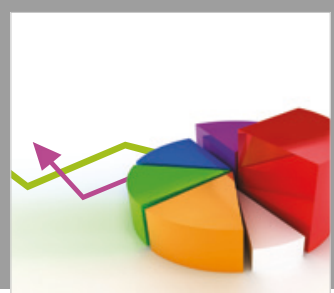

Journal of

Probability and Statistics
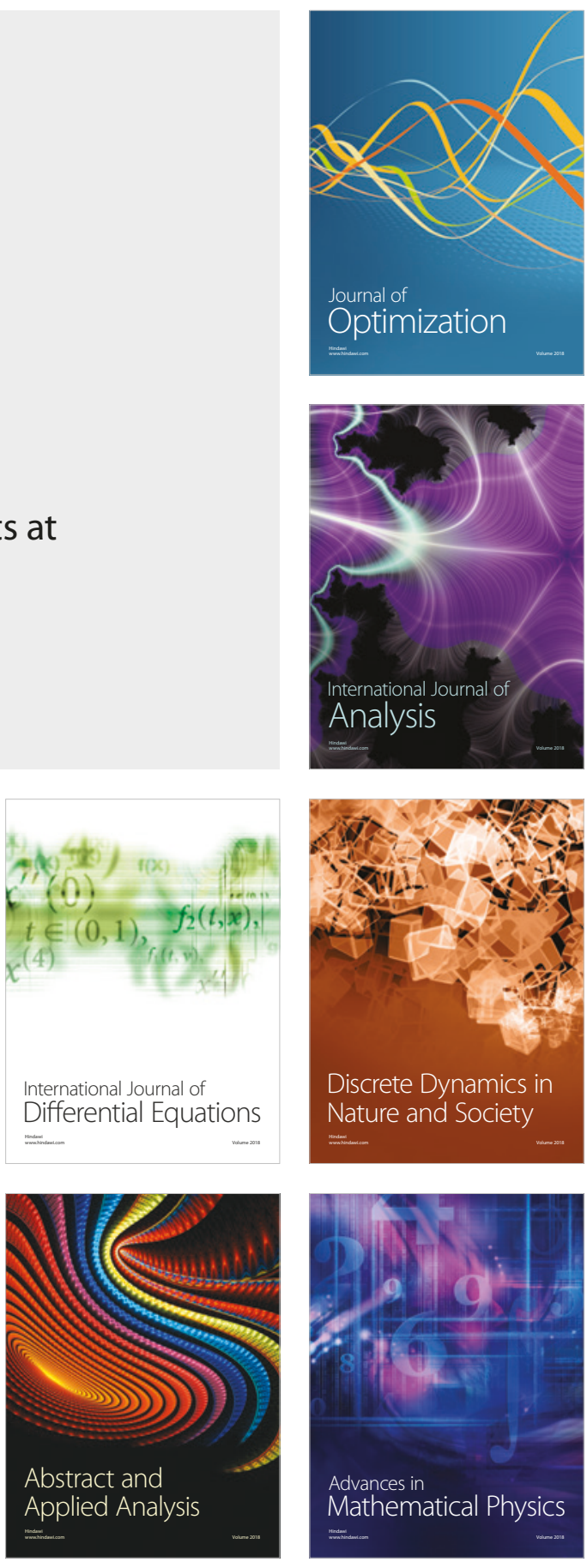